\title{
Inflammasome-mediated cell death in response to bacterial pathogens that access the host cell cytosol: lessons from Legionella pneumophila
}

\section{Cierra N. Casson and Sunny Shin*}

Department of Microbiology, Perelman School of Medicine, University of Pennsylvania, Philadelphia, PA, USA

\author{
Edited by: \\ Yongqun He, University of Michigan, \\ USA \\ Reviewed by: \\ Dario S. Zamboni, Universidade de \\ São Paulo, Brazil \\ Thomas Henry, Institut National de \\ la Santé et de la recherche \\ médicale, France \\ ${ }^{*}$ Correspondence: \\ Sunny Shin, Department of \\ Microbiology, Perelman School of \\ Medicine, University of \\ Pennsylvania, 3610 Hamilton Walk, \\ Philadelphia, PA 19104, USA \\ e-mail:sunshin@ \\ mail.med.upenn.edu
}

Cell death can be critical for host defense against intracellular pathogens because it eliminates a crucial replicative niche, and pro-inflammatory cell death can alert neighboring cells to the presence of pathogenic organisms and enhance downstream immune responses. Pyroptosis is a pro-inflammatory form of cell death triggered by the inflammasome, a multi-protein complex that assembles in the cytosol to activate caspase-1. Inflammasome activation by pathogens hinges upon violation of the host cell cytosol by activities such as the use of pore-forming toxins, the use of specialized secretion systems, or the cytosolic presence of the pathogen itself. Recently, a non-canonical inflammasome has been described that activates caspase-11 and also leads to pro-inflammatory cell death. Caspase-11 is activated rapidly and robustly in response to violation of the cytosol by bacterial pathogens as well. In this mini-review, we describe the canonical and non-canonical inflammasome pathways that are critical for host defense against a model intracellular bacterial pathogen that accesses the host cytosol_Legionella pneumophila.

Keywords: Legionella pneumophila, inflammasome, cell death, pyroptosis, caspase-11, caspase-1

\section{INTRODUCTION}

Cell death is an important innate immune effector mechanism to aid in clearance of intracellular pathogens, as it can eliminate a pathogen's replicative niche. Additionally, pro-inflammatory cell death can be critical for alerting neighboring cells to the presence of invading pathogens (Kono and Rock, 2008; Bergsbaken et al., 2009). The pro-inflammatory form of cell death known as pyroptosis is critical both for clearance of bacterial pathogens and for release of important proinflammatory cytokines in vivo (Fink and Cookson, 2005; Miao et al., 2010a). Pyroptosis is initiated in response to violation of the host cell cytosol by pathogenic microbes (Lamkanfi and Dixit, 2009). Violation of the cytosol can occur either by access via bacterial secretion systems, such as type IV (T4SS) or type III (T3SS) secretion systems, or by physical entry of a pathogen into the cytosol. Here, we discuss how cells induce proinflammatory cell death in response to microbes gaining cytosolic access by using Legionella pneumophila as a model intracellular pathogen.

\section{NOD-LIKE RECEPTORS RESPOND TO CYTOSOLIC ACCESS BY PATHOGENS}

Pattern recognition receptors (PRRs) are critical initiators of host defense against invading microorganisms (Janeway and Medzhitov, 2002; Medzhitov, 2007). Surface and endosomallyassociated PRRs, such as Toll-like receptors (TLRs), recognize pathogen-associated molecular patterns found in the extracellular space (Janeway and Medzhitov, 2002). However, many pathogenic organisms have mechanisms for accessing the host cytosol. Thus, many cells encode cytosolic PRRs, such as nucleotide-binding oligomerization domain (NOD)-like receptors (NLRs) (Harton et al., 2002), which act as guardians of cytosolic sanctity (Lamkanfi and Dixit, 2009). NLRs respond to "patterns of pathogenesis," such as membrane disruption, delivery of bacterial molecules into the host cytosol via specialized secretion systems, or pore-forming toxins, by activating the inflammasome (Fritz et al., 2006; Lamkanfi and Dixit, 2009; Vance et al., 2009; Davis et al., 2011; Franchi et al., 2012).

\section{CASPASE-1-DEPENDENT INFLAMMASOMES}

The canonical inflammasome is a multi-protein complex that assembles in the cytosol to activate the enzyme caspase-1, also known as interleukin-1 $\beta$ (IL-1 $1 \beta$ )-converting enzyme (ICE) (Martinon et al., 2002). Caspase-1 regulates secretion of IL-1 family cytokines and a pro-inflammatory form of cell death termed pyroptosis (Rathinam et al., 2012a). Caspase-1 processes IL-1 $\beta$ and IL-18 into their mature forms and aids in their secretion (Howard et al., 1991; Thornberry et al., 1992; Ghayur et al., 1997; Gu et al., 1997). Caspase-1 does not cleave IL-1 $\alpha$, though it can aid in IL-1 $\alpha$ secretion as well (Keller et al., 2008). IL1 family cytokines act in vivo to enhance immune responses against invading microorganisms (Labow et al., 1997; Bohn et al., 1998; Dinarello, 2009). Additionally, caspase-1-mediated pyroptosis enhances clearance of bacterial pathogens in vivo (Miao et al., 2010a).

NLRs respond to different stimuli when activating the inflammasome. Few NLRs have been shown to bind directly to their implicated substrates, and some are activated by a wide variety 
of stimuli. For example, NLRP3 responds to stimuli ranging from bacterial RNA to extracellular adenosine triphosphate and uric acid crystals (Kanneganti et al., 2006; Mariathasan et al., 2006; Martinon et al., 2006). Absent in melanoma 2 (AIM2) responds to the presence of cytosolic double-stranded DNA (Hornung et al., 2009; Roberts et al., 2009). In mice, ICE-protease activating factor (IPAF)/NLR family, CARD domain containing 4 (NLRC4) mediates inflammasome activation in response to three distinct stimuli-flagellin, the conserved inner rod component of the bacterial T3SS (PrgJ), and T3SS needle proteins (Franchi et al., 2006; Miao et al., 2006, 2010b; Lightfield et al., 2011; Yang et al., 2013). Biochemical studies have shown that the NLRs neuronal apoptosis inhibitory protein 5 (NAIP5) and NAIP6 coimmunoprecipitate with flagellin, while NAIP2 interacts specifically with PrgJ and NAIP1 interacts with the needle proteins (Kofoed and Vance, 2011; Zhao et al., 2011; Yang et al., 2013). NLRC4 appears to be an important adaptor for the NAIP receptors. The adaptor protein apoptosis-associated speck-like protein containing a carboxy-terminal caspase recruitment domain (ASC) often bridges the interaction between NLRs and caspase-1, allowing for oligomerization and auto-processing of caspase- 1 for activation (Srinivasula et al., 2002). Caspase-1 auto-processing is required for cytokine cleavage and secretion, though cell death can occur independently of caspase-1 proteolysis (Broz et al., 2010).

\section{THE NON-CANONICAL INFLAMMASOME}

Experiments examining inflammasome activation were first performed with macrophages from mice that lack caspase-1, and it was concluded that caspase-1 is solely responsible for inflammasome activation. However, the strain of mice used to generate the original caspase- 1 knockout has a caspase-11 polymorphism that eliminates protein expression. Thus, the original mice lack both caspase-1 and caspase-11 (Kuida et al., 1995; Kayagaki et al., 2011). Though it was reported that caspase-11 mediates septic shock in vivo, the cell-intrinsic role of caspase-11 in response to bacterial pathogens remained unclear (Wang et al., 1996, 1998). Recently, however, a non-canonical caspase-11dependent inflammasome has been described that contributes to IL- $1 \alpha$, IL-1 $\beta$, and IL-18 secretion and cell death in response to many Gram-negative bacteria. Caspase-11 is activated with delayed kinetics, taking 16-24h in vitro, in response to bacteria that do not typically access the host cell cytosol, such as non-pathogenic Escherichia coli (Kayagaki et al., 2011). For many Gram-negative bacteria, non-canonical inflammasome activation requires TIR-domain-containing adaptor-inducing interferon- $\beta$ (TRIF) and type I interferon (IFN) signaling downstream of TLR4 (Broz et al., 2012; Gurung et al., 2012; Rathinam et al., 2012b). Additionally, cytosolic lipopolysaccharide (LPS) activates caspase-11 independently of TLR4 (Hagar et al., 2013; Kayagaki et al., 2013). Pathogens that access or enter the host cytosol also induce non-canonical inflammasome activation, and this activation is more rapid than for other Gramnegative bacteria. One robust activator of the non-canonical inflammasome is the intracellular pathogen Legionella pneumophila (Aachoui et al., 2013; Case et al., 2013; Casson et al., 2013).

\section{Legionella pneumophila}

L. pneumophila is a Gram-negative bacterium that causes the severe pneumonia Legionnaires' disease (Fraser et al., 1977; McDade et al., 1977). L. pneumophila uses its dot/icm-encoded T4SS to translocate effector proteins into the host cytosol to establish an endoplasmic reticulum-derived vacuole that supports bacterial replication (Marra et al., 1992; Berger and Isberg, 1993; Sadosky et al., 1993; Roy et al., 1998; Segal et al., 1998; Vogel et al., 1998). The natural host for L. pneumophila is amoebae in aquatic reservoirs (Rowbotham, 1980; Fliermans et al., 1981), so while it has evolved to evade amoebic host defenses, it is not thought to have evolved to evade mammalian-specific immune responses. Therefore, as a consequence of accessing the host cytosol in mammalian cells, L. pneumophila triggers multiple pathways that elicit cell-intrinsic immune responses and induce cell death. These robust immune responses make the bacterium valuable for studying host defense against intracellular pathogens.

\section{L. pneumophila AND CASPASE-1-DEPENDENT INFLAMMASOME ACTIVATION}

It is well-understood that L. pneumophila triggers inflammasome activation and pyroptosis as a consequence of flagellin expression and T4SS activity (Figure 1). In murine macrophages, detection of flagellin by BIRC1e/NAIP5 mediates pyroptosis and contributes to restriction of $L$. pneumophila replication both in vitro and in vivo (Growney and Dietrich, 2000; Diez et al., 2003; Wright et al., 2003; Derré and Isberg, 2004; Zamboni et al., 2006; Kofoed and Vance, 2011; Zhao et al., 2011). Flagellin-deficient L. pneumophila ( $\triangle$ flaA Lp) evade NAIP5-mediated restriction and replicate in NAIP5-sufficient macrophages from C57BL/6 (B6) mice, in part because they do not induce as much caspase-1dependent cell death as wild-type (WT) Lp (Molofsky et al., 2006; Ren et al., 2006). NLRC4 also acts upstream of caspase-1 to induce flagellin-mediated restriction of replication, pore formation in the host membrane, and IL-1 $\beta$ release (Zamboni et al., 2006; Silveira and Zamboni, 2010). NLRC4 co-immunoprecipitates with NAIP5, consistent with the model that NLRC4 is an adaptor for NAIP5 (Zamboni et al., 2006; Kofoed and Vance, 2011; Zhao et al., 2011). The NAIP5/NLRC4-dependent cell death induced in B6 macrophages requires cytosolic access, as T4SS-deficient mutants $(\triangle \operatorname{dot} A \mathrm{Lp})$ do not activate the inflammasome. These data suggest that flagellin is translocated through the T4SS into the host cytosol during infection, though this has not been shown experimentally.

A/J mice express a hypomorphic allele of NAIP5 (Diez et al., 2000), and A/J macrophages still activate caspase-1 in response to WT Lp under certain infection conditions (Lamkanfi et al., 2007). However, using Naip5 $5^{-/-}$macrophages, it was shown that NAIP5 is required for caspase-1 activation in response to WT Lp (Lightfield et al., 2008). Interestingly, NAIP6 also interacts with L. pneumophila flagellin (Kofoed and Vance, 2011; Zhao et al., 2011). However, NAIP6 is insufficient for the restriction of L. pneumophila, as Naip5 $5^{-/}$macrophages and mice are permissive for infection (Lightfield et al., 2008), potentially due to lower expression levels of NAIP6 relative to NAIP5 in primary macrophages (Wright et al., 2003). NAIP5 and NLRC4 also 


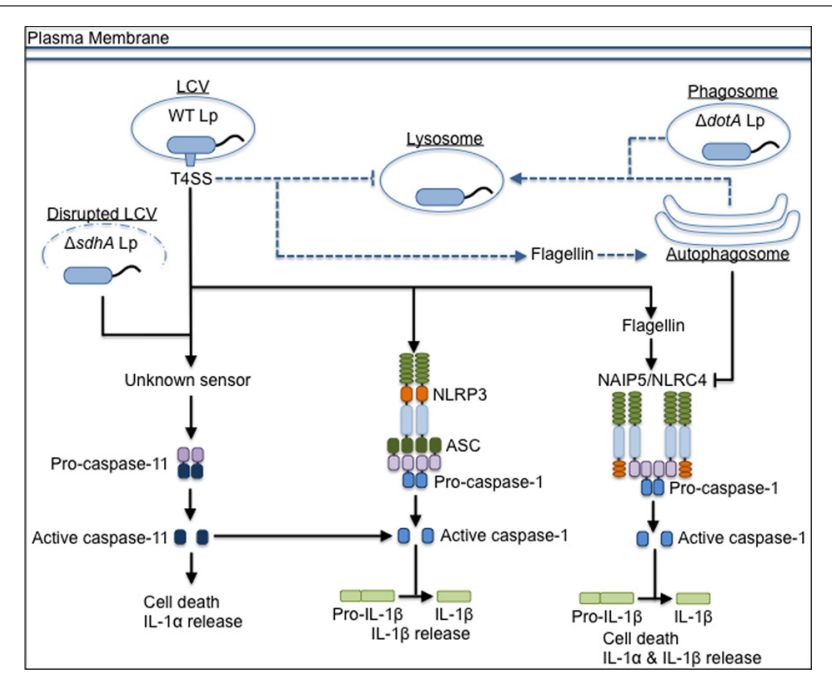

FIGURE 1 | The inflammasome-mediated response to $L$. pneumophila in murine macrophages. $L$. pneumophila that do not express a functional T4SS ( $\triangle$ dotA Lp) traffic to the lysosome, but wild-type L. pneumophila (WT $L p)$ use the T4SS to translocate effectors into the host cytosol to establish a replicative niche, the Legionella-containing vacuole (LCV), and block fusion with lysosomes. WT Lp triggers canonical caspase-1-dependent inflammasome activation through detection of translocated flagellin by NAIP5/NLRC4. NLRP3 and ASC contribute to IL-1 $\beta$ secretion in response to WT Lp. Detection of T4SS activity through an unknown sensor leads to caspase-11 activation, which contributes to inflammasome activation. Caspase-11 also responds to bacteria that aberrantly enter the cytosol ( $\triangle s d h A$ Lp) due to loss of LCV membrane integrity. Translocated flagellin triggers trafficking of WT Lp to the autophagosome, and induction of autophagy negatively regulates pyroptosis if there are low levels of flagellin in the host cell cytosol. Dashed lines represent vesicular trafficking patterns. Solid lines represent pathways for activation of the host response. Arrows at the end of lines represent induction, while flat bars at the end of lines represent inhibition.

contribute to the control of L. pneumophila replication by enhancing fusion of the Legionella-containing vacuole (LCV) with lysosomes during infections performed at a low multiplicity of infection (MOI) (Amer et al., 2006; Fortier et al., 2007). In addition, flagellin-dependent NLRC4 signaling leads to caspase-7-mediated restriction of $L$. pneumophila via enhanced lysosomal degradation of the bacterium (Akhter et al., 2009). NLRC4-mediated restriction in vivo is also partially caspase-1-independent through an unknown mechanism (Pereira et al., 2011). However, caspase-1 activation downstream of NLRC4 clearly induces pyroptosis and leads to IL-18 secretion both in vitro and in vivo, contributing to IFN- $\gamma$ production and the subsequent resolution of pulmonary infection (Brieland et al., 2000; Spörri et al., 2006; Archer et al., 2009; Case et al., 2009). Thus, the NAIP5/NLRC4 inflammasome may control L. pneumophila replication through multiple mechanisms. Further studies are needed to determine the relative contributions of these mechanisms.

Not surprisingly, infection conditions, including MOI, can affect the detection of caspase-1 activation in response to L. pneumophila, as higher MOIs likely enhance the number of macrophages that harbor bacteria. At higher MOIs, infection of B6 macrophages induces both NLRC4-dependent and NLRC4-independent inflammasome activation. NLRC4independent caspase- 1 activation and IL- $1 \beta$ and IL-18 secretion require ASC and NLRP3, although the identity of the L. pneumophila-derived signal sensed via NLRP3 is unknown (Case et al., 2009, 2013; Casson et al., 2013). Caspase-1 cleavage in the absence of ASC can be detected in either the supernatant or the cytosol, depending on the MOI (Case et al., 2009; Abdelaziz et al., 2011a). ASC also drives formation of a punctate structure containing caspase- 1 and NLRC4 in L. pneumophila-infected macrophages (Case and Roy, 2011). At early timepoints, pore formation is not observed in the absence of NLRC4, though cell death still occurs in the absence of ASC. Recruitment of NLRC4 into the ASC complex appears to dampen NLRC4 activity because pyroptosis occurs at a higher rate in the absence of ASC (Case and Roy, 2011). Further studies are needed to elucidate the temporal and spatial coordination of the ASC- and NLRC4-dependent inflammasomes and how they are triggered by L. pneumophila.

\section{INFLAMMASOME ACTIVATION IN HUMAN CELLS}

Unlike macrophages from most inbred mouse strains, human cells are permissive for $L$. pneumophila replication. The mechanisms underlying inflammasome-mediated control of L. pneumophila replication in human cells are unclear. Humans express only one homolog of the numerous murine NAIP paralogs (Scharf et al., 1996). The homolog, human NAIP (hNAIP), restricts growth of WT Lp (Vinzing et al., 2008). Additionally, the human NLRC4 homolog, human IPAF (hIPAF), also restricts L. pneumophila replication. Overexpression of full-length hNAIP in HEK293T cells increases cell death in response to L. pneumophila (Boniotto et al., 2012), and overexpression of hNAIP in the murine macrophage RAW264.7 cell line mediates flagellininduced pyroptosis and IL-1 $\beta$ secretion (Katagiri et al., 2012), suggesting that it may function similarly to NAIP5. However, unlike NAIP5, hNAIP does not co-immunoprecipitate with flagellin and instead interacts with T3SS needle proteins (Zhao et al., 2011; Yang et al., 2013). Thus, it is unclear whether hNAIP senses flagellin or another L. pneumophila-derived ligand, how hNAIP restricts $L$. pneumophila replication, and if hNAIP contributes to cell death or IL- $1 \beta$ secretion in primary human cells.

The implication that the IPAF/NAIP/caspase-1 inflammasome contributes to restriction of $L$. pneumophila is pervasive, though caspase-1 activation in response to L. pneumophila has not been explicitly shown in primary cells from humans, a naturally susceptible host. Immortalized human alveolar epithelial cells activate caspase-1 in response to L. pneumophila, though primary human monocytes and monocyte-derived macrophages (MDMs) do not produce detectable levels of processed or active caspase-1 (Santic et al., 2007; Furugen et al., 2008; Abdelaziz et al., 2011b). Additionally, the expression of ASC is moderately down-regulated in infected monocytes, potentially contributing to evasion of inflammasome activation in human cells by $L$. pneumophila (Abdelaziz et al., 2011b). Future studies in primary MDMs and human alveolar macrophages are needed to clarify the role of the inflammasome in restricting $L$. pneumophila replication in human cells (Figure 2). 


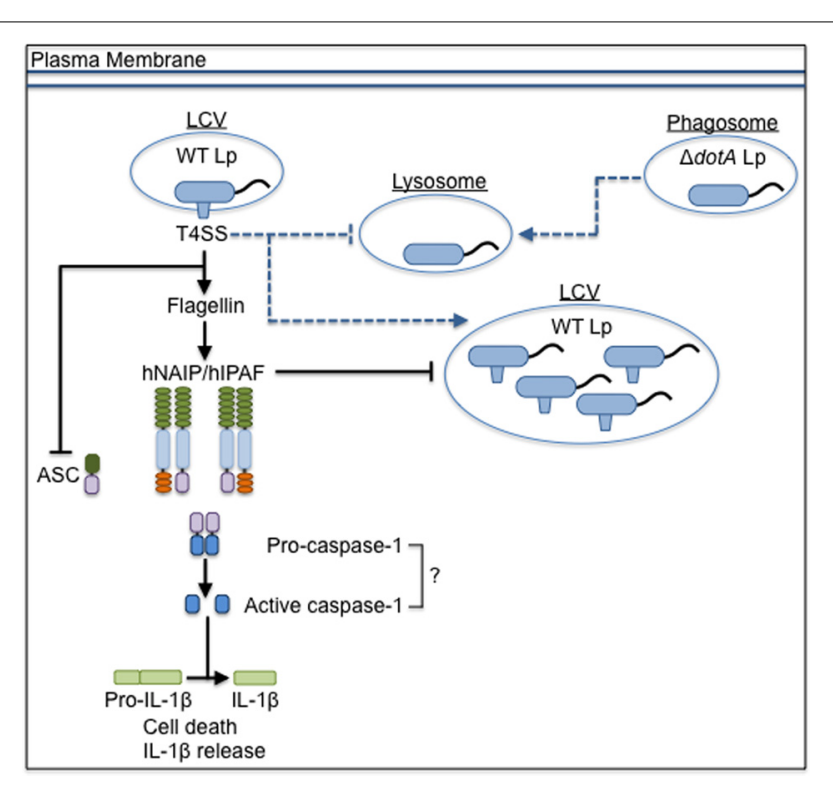

FIGURE 2 | The inflammasome-mediated response to $L$. pneumophila in human cells. $L$. pneumophila that do not express a functional T4SS ( $\triangle$ dotA Lp) traffic to the lysosome, but wild-type L. pneumophila (WT Lp) use the T4SS to translocate effectors into the host cytosol to establish a replicative niche, the Legionella-containing vacuole (LCV), and block fusion with lysosomes. The presence of flagellin triggers signaling through hNAIP/hIPAF that blocks replication of WT Lp, though it is unclear if caspase-1 is involved in restriction. T4SS activity down-regulates the expression of ASC. Dashed lines represent vesicular trafficking patterns. Solid lines represent pathways for activation of the host response. Arrows at the end of lines represent induction, while flat bars at the end of lines represent inhibition.

\section{INFLAMMASOME ACTIVATION AND AUTOPHAGY}

In murine macrophages, autophagy is induced shortly after phagocytosis of L. pneumophila, as components of the autophagy pathway co-localize with the LCV (Amer and Swanson, 2005). LCVs in A/J macrophages show delayed autophagosome maturation compared to LCVs in B6 macrophages, potentially contributing to increased replication of the bacterium. When expression of the autophagy component ATG5 is silenced, L. pneumophila replication in A/J macrophages increases. Additionally, replication of L. pneumophila decreases slightly when autophagy is induced exogenously, suggesting that autophagy contributes to restriction of L. pneumophila replication (Matsuda et al., 2009). Under low MOI infection conditions where there is minimal induction of pyroptosis, it was revealed that the induction of autophagy dampens pyroptosis in response to L. pneumophila, and turnover of autophagosomes requires NAIP5, NLRC4, and caspase-1 (Byrne et al., 2013). Collectively, these data suggest that NAIP5 inflammasome activation contributes to the restriction of L. pneumophila replication by inducing autophagy and/or pyroptosis, depending on the MOI and amount of flagellin present. How competing host and bacterial factors influence the outcome of inflammasome activation and autophagy during infection remains unclear and may be clarified by studies examining the temporal regulation of inflammasome activation and autophagy at a single-cell level.

\section{L. pneumophila AND NON-CANONICAL INFLAMMASOME ACTIVATION}

Though $\triangle$ flaA Lp avoid NAIP5/NLRC4-mediated pyroptosis and can replicate in $\mathrm{B} 6$ macrophages, $\triangle$ flaA Lp trigger an additional form of cell death. Recently, caspase-11 has been shown to contribute to inflammasome activation in response to L. pneumophila (Figure 1). After MyD88 and TRIF-dependent upregulation of caspase-11, host cells undergo rapid caspase-11-mediated cell death, occurring in less than $4 \mathrm{~h}$, in response to $\Delta$ flaA Lp (Case et al., 2013; Casson et al., 2013). Non-canonical inflammasome activation in response to $\triangle$ flaA Lp requires T4SS-mediated cytosolic access, as $\Delta \operatorname{dot} A \mathrm{Lp}$ do not activate caspase-11. Like caspase-1-mediated pyroptosis, caspase-11-dependent cell death leads to release of important inflammatory mediators, such as IL- $1 \alpha$, IL- $1 \beta$, and IL-18. Caspase-11 is required for cell death and IL- $1 \alpha$ release and additionally enhances NLRP3-dependent caspase- 1 activation and IL- $1 \beta$ and IL-18 secretion (Case et al., 2013; Casson et al., 2013). IL-1 $\alpha$ release in vivo is critical for host defense, including neutrophil recruitment to the airway space and control of bacterial burden, though there are caspase-11-independent sources of IL-1 $\alpha$ in vivo as well (Barry et al., 2013; Casson et al., 2013). Caspase-11 also contributes to NAIP5/NLRC4-mediated inflammasome activation and restricts WT Lp by enhancing phago-lysosomal fusion (Akhter et al., 2012). In its non-lytic role, caspase-11 modulates actin polymerization and phosphorylation of cofilin to promote lysosomal trafficking of pathogenic, but not non-pathogenic, bacteria. Additionally, caspase-11 contributes to control of WT Lp replication in vivo (Akhter et al., 2012).

Caspase-11 responds not only to vacuolar bacteria that access the host cytosol through the T4SS but also to L. pneumophila that escape from the vacuole and aberrantly enter the cytosol (Aachoui et al., 2013). The T4SS-translocated effector SdhA is critical for bacterial growth in primary macrophages (Laguna et al., 2006; Liu et al., 2008). Macrophages infected with $\Delta s d h A$ Lp undergo cell death because SdhA is required to maintain LCV membrane integrity (Creasey and Isberg, 2012). Therefore, $\Delta s d h A$ Lp aberrantly enter the host cytosol where they become degraded, induce type I IFN, and activate caspase-1 via AIM2 (Monroe et al., 2009; Creasey and Isberg, 2012; Ge et al., 2012). In addition, $\Delta s d h A$ Lp induce rapid caspase-11-dependent cell death independently of bacterial flagellin (Aachoui et al., 2013). It appears that AIM2 responds to cytosolic L. pneumophila by producing IL-1 $\beta$, whereas caspase-11 mediates cell death. However, L. pneumophila does not normally enter the cytosol, so the upstream mediators of caspase-11 activation may be different for $\Delta s d h A \Delta$ flaA bacteria that enter the cytosol and $\triangle$ flaA bacteria that remain within the vacuole. Whether the bacteria physically enter the cytosol or not, these unique pathways upstream of caspase-11 are likely relevant for defense against other pathogens that lack or down-regulate flagellin during infection.

Non-canonical inflammasome activation is a recently described phenomenon, so there are many questions that remain unanswered. Currently, no NLRs have been identified that act upstream of caspase-11 to induce non-canonical inflammasome activation. As L. pneumophila rapidly and robustly activates caspase-11, it will be a valuable tool for future studies aiming 
to identify NLRs or other host factors critical for caspase-11 activation. The only bacterial factor that has been shown to initiate non-canonical inflammasome activation is cytosolic LPS (Hagar et al., 2013; Kayagaki et al., 2013). For some Gramnegative bacteria, it is thought that bacterial RNA may access the host cytosol to activate NLRP3 and caspase-11 (Kanneganti et al., 2006; Rathinam et al., 2012b). However, translocation of L. pneumophila RNA to initiate inflammasome activation has not been verified experimentally. Additionally, though cytosolic LPS may trigger caspase-11 during infection with $\Delta s d h A$ Lp that aberrantly enter the cytosol, it is unclear if LPS is sensed by host cells to initiate non-canonical inflammasome activation in the context of infection with L. pneumophila that remain within the LCV. Further studies are needed to clarify what triggers the host response to $\triangle$ flaA Lp and to elucidate the molecular pathways that lead to caspase-11-mediated cell death.

\section{CONCLUDING REMARKS}

Studying the inflammasome pathways triggered by the pathogen L. pneumophila has shaped our knowledge of how host cells are poised to respond to violation by intracellular pathogens. Whether the bacterium utilizes its T4SS to access the host cytosol, additionally delivers flagellin into the cytoplasm, or physically enters the cytosol itself, the host has evolved multiple ways to restrict replication of the pathogen and trigger immunity.

\section{ACKNOWLEDGMENTS}

Work in our lab is supported by National Institutes of Health grant AI087963, American Lung Association grant RG-268528N, American Heart Association grant 13BGIA14780070, and the University of Pennsylvania University Research Foundation (SS). CNC is supported by the National Science Foundation under Grant No. DGE-0822 (graduate research fellowship).

\section{REFERENCES}

Aachoui, Y., Leaf, I. A., Hagar, J. A., Fontana, M. F., Campos, C. G., Zak, D. E., et al. (2013). Caspase-11 protects against bacteria that escape the vacuole. Science 339, 975-978. doi: 10.1126/science.1230751

Abdelaziz, D. H. A., Gavrilin, M. A., Akhter, A., Caution, K., Kotrange, S., Khweek, A. A., et al. (2011a). Asc-dependent and independent mechanisms contribute to restriction of Legionella pneumophila infection in murine macrophages. Front. Microbiol. 2:18. doi: 10.3389/fmicb.2011.00018

Abdelaziz, D. H., Gavrilin, M. A., Akhter, A., Caution, K., Kotrange, S., Khweek, A. A., et al. (2011b). Apoptosis-associated speck-like protein (ASC) controls Legionella pneumophila infection in human monocytes. J. Biol. Chem. 286, 3203-3208. doi: 10.1074/jbc.M110.197681

Akhter, A., Caution, K., Abu Khweek, A., Tazi, M., Abdulrahman, B. A., Abdelaziz, D. H. A., et al. (2012). Caspase-11 promotes the fusion of phagosomes harboring pathogenic bacteria with lysosomes by modulating actin polymerization. Immunity 37, 35-47. doi: 10.1016/j.immuni.2012.05.001

Akhter, A., Gavrilin, M. A., Frantz, L., Washington, S., Ditty, C., Limoli, D., et al. (2009). Caspase-7 activation by the Nlrc4/Ipaf inflammasome restricts Legionella pneumophila infection. PLoS Pathog. 5:e1000361. doi: 10.1371/journal.ppat.1000361

Amer, A., Franchi, L., Kanneganti, T.-D., Body-Malapel, M., Ozören, N., Brady, G., et al. (2006). Regulation of Legionella phagosome maturation and infection through flagellin and host Ipaf. J. Biol. Chem. 281, 35217-35223. doi: 10.1074/jbc.M604933200

Amer, A. O., and Swanson, M. S. (2005). Autophagy is an immediate macrophage response to Legionella pneumophila. Cell. Microbiol. 7, 765-778. doi: 10.1111/j.1462-5822.2005.00509.x
Archer, K. A., Alexopoulou, L., Flavell, R. A., and Roy, C. R. (2009). Multiple MyD88-dependent responses contribute to pulmonary clearance of Legionella pneumophila. Cell. Microbiol. 11, 21-36. doi: 10.1111/j.1462-5822.2008. 01234.x

Barry, K. C., Fontana, M. F., Portman, J. L., Dugan, A. S., and Vance, R. E. (2013). IL- $1 \alpha$ signaling initiates the inflammatory response to virulent Legionella pneumophila in vivo. J. Immunol. 190, 6329-6339. doi: 10.4049/jimmunol. 1300100

Berger, K. H., and Isberg, R. R. (1993). Two distinct defects in intracellular growth complemented by a single genetic locus in Legionella pneumophila. Mol. Microbiol. 7, 7-19. doi: 10.1111/j.1365-2958.1993.tb01092.x

Bergsbaken, T., Fink, S. L., and Cookson, B. T. (2009). Pyroptosis: host cell death and inflammation. Nat. Rev. Microbiol. 7, 99-109. doi: 10.1038/nrmicro2070

Bohn, E., Sing, A., Zumbihl, R., Bielfeldt, C., Okamura, H., Kurimoto, M., et al. (1998). IL-18 (IFN-gamma-inducing factor) regulates early cytokine production in, and promotes resolution of, bacterial infection in mice. J. Immunol. 160, 299-307.

Boniotto, M., Tailleux, L., Lomma, M., Gicquel, B., Buchrieser, C., Garcia, S., et al. (2012). Population variation in NAIP functional copy number confers increased cell death upon Legionella pneumophila infection. Hum. Immunol. 73, 196-200. doi: 10.1016/j.humimm.2011.10.014

Brieland, J. K., Jackson, C., Hurst, S., Loebenberg, D., Muchamuel, T., Debets, R., et al. (2000). Immunomodulatory role of endogenous interleukin-18 in gamma interferon-mediated resolution of replicative Legionella pneumophila lung infection. Infect. Immun. 68, 6567-6573. doi: 10.1128/IAI.68.12.6567-6573.2000

Broz, P., Ruby, T., Belhocine, K., Bouley, D. M., Kayagaki, N., Dixit, V. M., et al. (2012). Caspase-11 increases susceptibility to Salmonella infection in the absence of caspase-1. Nature 490, 288-291. doi: 10.1038/nature11419

Broz, P., von Moltke, J., Jones, J. W., Vance, R. E., and Monack, D. M. (2010). Differential requirement for Caspase-1 autoproteolysis in pathogeninduced cell death and cytokine processing. Cell Host Microbe 8, 471-483. doi: 10.1016/j.chom.2010.11.007

Byrne, B. G., Dubuisson, J.-F., Joshi, A. D., Persson, J. J., and Swanson, M. S. (2013). Inflammasome components coordinate autophagy and pyroptosis as macrophage responses to infection. mBio 4:e00620-12. doi: 10.1128/mBio.00620-12

Case, C. L., Kohler, L. J., Lima, J. B., Strowig, T., de Zoete, M. R., Flavell, R. A., et al. (2013). Caspase-11 stimulates rapid flagellin-independent pyroptosis in response to Legionella pneumophila. Proc. Natl. Acad. Sci. U.S.A. 110, 1851-1856. doi: 10.1073/pnas.1211521110

Case, C. L., and Roy, C. R. (2011). Asc modulates the function of NLRC4 in response to infection of macrophages by Legionella pneumophila. mBio 2:e00117-11. doi: 10.1128/mBio.00117-11

Case, C. L., Shin, S., and Roy, C. R. (2009). Asc and Ipaf Inflammasomes direct distinct pathways for caspase-1 activation in response to Legionella pneumophila. Infect. Immun. 77, 1981-1991. doi: 10.1128/IAI.01382-08

Casson, C. N., Copenhaver, A. M., Zwack, E. E., Nguyen, H. T., Strowig, T., Javdan, B., et al. (2013). Caspase-11 activation in response to bacterial secretion systems that access the host cytosol. PLoS Pathog. 9:e1003400. doi: 10.1371/journal.ppat. 1003400

Creasey, E. A., and Isberg, R. R. (2012). The protein SdhA maintains the integrity of the Legionella-containing vacuole. Proc. Natl. Acad. Sci. U.S.A. 109, 3481-3486. doi: 10.1073/pnas.1121286109

Davis, B. K., Wen, H., and Ting, J. P. Y. (2011). The inflammasome NLRs in immunity, inflammation, and associated diseases. Annu. Rev. Immunol. 29, 707-735. doi: 10.1146/annurev-immunol-031210-101405

Derré, I., and Isberg, R. R. (2004). Macrophages from mice with the restrictive Lgn1 allele exhibit multifactorial resistance to Legionella pneumophila. Infect. Immun. 72, 6221-6229. doi: 10.1128/IAI.72.11.6221-6229.2004

Diez, E., Lee, S.-H., Gauthier, S., Yaraghi, Z., Tremblay, M., Vidal, S., et al. (2003). Bircle is the gene within the Lgn 1 locus associated with resistance to Legionella pneumophila. Nat. Genet. 33, 55-60. doi: 10.1038/ng1065

Diez, E., Yaraghi, Z., MacKenzie, A., and Gros, P. (2000). The neuronal apoptosis inhibitory protein (Naip) is expressed in macrophages and is modulated after phagocytosis and during intracellular infection with Legionella pneumophila. J. Immunol. 164, 1470-1477.

Dinarello, C. A. (2009). Immunological and inflammatory functions of the interleukin-1 family. Annu. Rev. Immunol. 27, 519-550. doi: 10.1146/annurev.immunol.021908.132612 
Fink, S. L., and Cookson, B. T. (2005). Apoptosis, pyroptosis, and necrosis: mechanistic description of dead and dying eukaryotic cells. Infect. Immun. 73, 1907-1916. doi: 10.1128/IAI.73.4.1907-1916.2005

Fliermans, C. B., Cherry, W. B., Orrison, L. H., Smith, S. J., Tison, D. L., and Pope, D. H. (1981). Ecological distribution of Legionella pneumophila. Appl. Environ. Microbiol. 41, 9-16.

Fortier, A., de Chastellier, C., Balor, S., and Gros, P. (2007). Bircle/Naip5 rapidly antagonizes modulation of phagosome maturation by Legionella pneumophila. Cell. Microbiol. 9, 910-923. doi: 10.1111/j.1462-5822.2006.00839.x

Franchi, L., Amer, A., Body-Malapel, M., Kanneganti, T.-D., Ozören, N., Jagirdar, R., et al. (2006). Cytosolic flagellin requires Ipaf for activation of caspase-1 and interleukin lbeta in salmonella-infected macrophages. Nat. Immunol. 7, 576-582. doi: 10.1038/ni1346

Franchi, L., Muñoz-Planillo, R., and Núñez, G. (2012). Sensing and reacting to microbes through the inflammasomes. Nat. Immunol. 13, 325-332. doi: 10.1038/ni.2231

Fraser, D. W., Tsai, T. R., Orenstein, W., Parkin, W. E., Beecham, H. J., Sharrar, R. G., et al. (1977). Legionnaires' disease: description of an epidemic of pneumonia. N. Engl. J. Med. 297, 1189-1197. doi: 10.1056/NEJM197712012972201

Fritz, J. H., Ferrero, R. L., Philpott, D. J., and Girardin, S. E. (2006). Nod-like proteins in immunity, inflammation and disease. Nat. Immunol. 7, 1250-1257. doi: $10.1038 / \mathrm{ni} 1412$

Furugen, M., Higa, F., Hibiya, K., Teruya, H., Akamine, M., Haranaga, S., et al. (2008). Legionella pneumophila infection induces programmed cell death, caspase activation, and release of high-mobility group box 1 protein in A549 alveolar epithelial cells: inhibition by methyl prednisolone. Respir. Res. 9, 39. doi: 10.1186/1465-9921-9-39

Ge, J., Gong, Y.-N., Xu, Y., and Shao, F. (2012). Preventing bacterial DNA release and absent in melanoma 2 inflammasome activation by a Legionella effector functioning in membrane trafficking. Proc. Natl. Acad. Sci. U.S.A. 109, 6193-6198. doi: 10.1073/pnas.1117490109

Ghayur, T., Banerjee, S., Hugunin, M., Butler, D., Herzog, L., Carter, A., et al. (1997). Caspase-1 processes IFN-gamma-inducing factor and regulates LPSinduced IFN-gamma production. Nature 386, 619-623. doi: 10.1038/386619a0

Growney, J. D., and Dietrich, W. F. (2000). High-resolution genetic and physical map of the Lgn1 interval in C57BL/6J implicates Naip2 or Naip5 in Legionella pneumophila pathogenesis. Genome Res. 10, 1158-1171. doi: 10.1101/gr.10.8.1158

Gu, Y., Kuida, K., Tsutsui, H., Ku, G., Hsiao, K., Fleming, M. A., et al. (1997). Activation of interferon-gamma inducing factor mediated by interleukin-1beta converting enzyme. Science 275, 206-209. doi: 10.1126/science.275.5297.206

Gurung, P., Malireddi, R. K. S., Anand, P. K., Demon, D., Walle, L. V., Liu, Z., et al. (2012). TRIF-mediated caspase-11 production integrates TLR4- and Nlrp3 inflammasome-mediated host defense against enteropathogens. J. Biol. Chem. 287, 34474-34483. doi: 10.1074/jbc.M112.401406

Hagar, J. A., Powell, D. A., Aachoui, Y., Ernst, R. K., and Miao, E. A. (2013). Cytoplasmic LPS activates caspase-11: implications in TLR4-independent endotoxic shock. Science 341, 1250-1253. doi: 10.1126/science. 1240988

Harton, J. A., Linhoff, M. W., Zhang, J., and Ting, J. P. Y. (2002). Cutting edge: CATERPILLER: a large family of mammalian genes containing CARD, pyrin, nucleotide-binding, and leucine-rich repeat domains. J. Immunol. 169, 4088-4093.

Hornung, V., Ablasser, A., Charrel-Dennis, M., Bauernfeind, F., Horvath, G., Caffrey, D. R., et al. (2009). AIM2 recognizes cytosolic dsDNA and forms a caspase-1-activating inflammasome with ASC. Nature 458, 514-518. doi: 10.1038 /nature 07725

Howard, A. D., Kostura, M. J., Thornberry, N., Ding, G. J., Limjuco, G., Weidner, J., et al. (1991). IL-1-converting enzyme requires aspartic acid residues for processing of the IL-1 beta precursor at two distinct sites and does not cleave $31-\mathrm{kDa}$ IL-1 alpha. J. Immunol. 147, 2964-2969.

Janeway, C. A., and Medzhitov, R. (2002). Innate immune recognition. Annu. Rev. Immunol. 20, 197-216. doi: 10.1146/annurev.immunol.20.083001.084359

Kanneganti, T.-D., Ozören, N., Body-Malapel, M., Amer, A., Park, J.-H., Franchi, L., et al. (2006). Bacterial RNA and small antiviral compounds activate caspase-1 through cryopyrin/Nalp3. Nature 440, 233-236. doi: 10.1038/nature04517

Katagiri, N., Shobuike, T., Chang, B., Kukita, A., and Miyamoto, H. (2012). The human apoptosis inhibitor NAIP induces pyroptosis in macrophages infected with Legionella pneumophila. Microbes Infect. 14, 1123-1132. doi: 10.1016/j.micinf.2012.03.006
Kayagaki, N., Warming, S., Lamkanfi, M., Vande Walle, L., Louie, S., Dong, J., et al. (2011). Non-canonical inflammasome activation targets caspase-11. Nature 479, 117-121. doi: 10.1038/nature 10558

Kayagaki, N., Wong, M. T., Stowe, I. B., Ramani, S. R., Gonzalez, L. C., Akashi-Takamura, S., et al. (2013). Noncanonical inflammasome activation by intracellular LPS independent of TLR4. Science 341, 1246-1249. doi: 10.1126/science. 1240248

Keller, M., Rüegg, A., Werner, S., and Beer, H.-D. (2008). Active caspase-1 is a regulator of unconventional protein secretion. Cell 132, 818-831. doi: 10.1016/j.cell.2007.12.040

Kofoed, E. M., and Vance, R. E. (2011). Innate immune recognition of bacterial ligands by NAIPs determines inflammasome specificity. Nature 477, 592-595. doi: 10.1038/nature10394

Kono, H., and Rock, K. L. (2008). How dying cells alert the immune system to danger. Nat. Rev. Immunol. 8, 279-289. doi: 10.1038/nri2215

Kuida, K., Lippke, J. A., Ku, G., Harding, M. W., Livingston, D. J., Su, M. S., et al. (1995). Altered cytokine export and apoptosis in mice deficient in interleukin-1 beta converting enzyme. Science 267, 2000-2003. doi: 10.1126/science.7535475

Labow, M., Shuster, D., Zetterstrom, M., Nunes, P., Terry, R., Cullinan, E. B., et al. (1997). Absence of IL-1 signaling and reduced inflammatory response in IL-1 type I receptor-deficient mice. J. Immunol. 159, 2452-2461.

Laguna, R. K., Creasey, E. A., Li, Z., Valtz, N., and Isberg, R. R. (2006). A Legionella pneumophila-translocated substrate that is required for growth within macrophages and protection from host cell death. Proc. Natl. Acad. Sci. U.S.A. 103, 18745-18750. doi: 10.1073/pnas.0609012103

Lamkanfi, M., Amer, A., Kanneganti, T.-D., Muñoz-Planillo, R., Chen, G., Vandenabeele, P., et al. (2007). The Nod-like receptor family member Naip5/Bircle restricts Legionella pneumophila growth independently of caspase1 activation. J. Immunol. 178, 8022-8027.

Lamkanfi, M., and Dixit, V. M. (2009). Inflammasomes: guardians of cytosolic sanctity. Immunol. Rev. 227, 95-105. doi: 10.1111/j.1600-065X.2008.00730.x

Lightfield, K. L., Persson, J., Brubaker, S. W., Witte, C. E., von Moltke, J., Dunipace, E. A., et al. (2008). Critical function for Naip5 in inflammasome activation by a conserved carboxy-terminal domain of flagellin. Nat. Immunol. 9, 1171-1178. doi: 10.1038/ni.1646

Lightfield, K. L., Persson, J., Trinidad, N. J., Brubaker, S. W., Kofoed, E. M., Sauer, J.D., et al. (2011). Differential requirements for NAIP5 in activation of the NLRC4 inflammasome. Infect. Immun. 79, 1606-1614. doi: 10.1128/IAI.01187-10

Liu, Y., Gao, P., Banga, S., and Luo, Z.-Q. (2008). An in vivo gene deletion system for determining temporal requirement of bacterial virulence factors. Proc. Natl. Acad. Sci. U.S.A. 105, 9385-9390. doi: 10.1073/pnas.0801055105

Mariathasan, S., Weiss, D. S., Newton, K., McBride, J., O’Rourke, K., Roose-Girma, M., et al. (2006). Cryopyrin activates the inflammasome in response to toxins and ATP. Nature 440, 228-232. doi: 10.1038/nature04515

Marra, A., Blander, S. J., Horwitz, M. A., and Shuman, H. A. (1992). Identification of a Legionella pneumophila locus required for intracellular multiplication in human macrophages. Proc. Natl. Acad. Sci. U.S.A. 89, 9607-9611. doi: 10.1073/pnas.89.20.9607

Martinon, F., Burns, K., and Tschopp, J. (2002). The inflammasome: a molecular platform triggering activation of inflammatory caspases and processing of proIL-beta. Mol. Cell 10, 417-426. doi: 10.1016/S1097-2765(02)00599-3

Martinon, F., Pétrilli, V., Mayor, A., Tardivel, A., and Tschopp, J. (2006). Goutassociated uric acid crystals activate the NALP3 inflammasome. Nature 440, 237-241. doi: 10.1038/nature04516

Matsuda, F., Fujii, J., and Yoshida, S.-I. (2009). Autophagy induced by 2-deoxy-Dglucose suppresses intracellular multiplication of Legionella pneumophila in A/J mouse macrophages. Autophagy 5, 484-493. doi: 10.4161/auto.5.4.7760

McDade, J. E., Shepard, C. C., Fraser, D. W., Tsai, T. R., Redus, M. A., and Dowdle, W. R. (1977). Legionnaires' disease: isolation of a bacterium and demonstration of its role in other respiratory disease. N. Engl. J. Med. 297, 1197-1203. doi 10.1056/NEJM197712012972202

Medzhitov, R. (2007). Recognition of microorganisms and activation of the immune response. Nature 449, 819-826. doi: 10.1038/nature06246

Miao, E. A., Alpuche-Aranda, C. M., Dors, M., Clark, A. E., Bader, M. W., Miller, S. I., et al. (2006). Cytoplasmic flagellin activates caspase-1 and secretion of interleukin 1beta via Ipaf. Nat. Immunol. 7, 569-575. doi: 10.1038/ ni1344

Miao, E. A., Leaf, I. A., Treuting, P. M., Mao, D. P., Dors, M., Sarkar, A., et al. (2010a). Caspase-1-induced pyroptosis is an innate immune effector 
mechanism against intracellular bacteria. Nat. Immunol. 11, 1136-1142. doi: 10.1038/ni.1960

Miao, E. A., Mao, D. P., Yudkovsky, N., Bonneau, R., Lorang, C. G., Warren, S. E., et al. (2010b). Innate immune detection of the type III secretion apparatus through the NLRC4 inflammasome. Proc. Natl. Acad. Sci. U.S.A. 107, 3076-3080. doi: 10.1073/pnas.0913087107

Molofsky, A. B., Byrne, B. G., Whitfield, N. N., Madigan, C. A., Fuse, E. T., Tateda, K., et al. (2006). Cytosolic recognition of flagellin by mouse macrophages restricts Legionella pneumophila infection. J. Exp. Med. 203, 1093-1104. doi: 10.1084/jem.20051659

Monroe, K. M., McWhirter, S. M., and Vance, R. E. (2009). Identification of host cytosolic sensors and bacterial factors regulating the type I interferon response to Legionella pneumophila. PLoS Pathog. 5:e1000665. doi: 10.1371/journal.ppat.1000665

Pereira, M. S. F., Morgantetti, G. F., Massis, L. M., Horta, C. V., Hori, J. I., and Zamboni, D. S. (2011). Activation of NLRC4 by flagellated bacteria triggers caspase-1-dependent and -independent responses to restrict Legionella pneumophila replication in macrophages and in vivo. J. Immunol. 187, 6447-6455. doi: 10.4049/jimmunol.1003784

Rathinam, V. A. K., Vanaja, S. K., and Fitzgerald, K. A. (2012a). Regulation of inflammasome signaling. Nat. Immunol. 13, 333-332. doi: 10.1038/ni.2237

Rathinam, V. A. K., Vanaja, S. K., Waggoner, L., Sokolovska, A., Becker, C., Stuart, L. M., et al. (2012b). TRIF licenses Caspase-11-dependent NLRP3 inflammasome activation by gram-negative bacteria. Cell 150, 606-619. doi: 10.1016/j.cell.2012.07.007

Ren, T., Zamboni, D. S., Roy, C. R., Dietrich, W. F., and Vance, R. E. (2006). Flagellin-deficient Legionella mutants evade caspase-1- and Naip5-mediated macrophage immunity. PLoS Pathog. 2:e18. doi: 10.1371/journal.ppat.0020018

Roberts, T. L., Idris, A., Dunn, J. A., Kelly, G. M., Burnton, C. M., Hodgson, S., et al. (2009). HIN-200 proteins regulate caspase activation in response to foreign cytoplasmic DNA. Science 323, 1057-1060. doi: 10.1126/science.1169841

Rowbotham, T. J. (1980). Preliminary report on the pathogenicity of Legionella pneumophila for freshwater and soil amoebae. J. Clin. Pathol. 33, 1179-1183. doi: $10.1136 /$ jcp.33.12.1179

Roy, C. R., Berger, K. H., and Isberg, R. R. (1998). Legionella pneumophila DotA protein is required for early phagosome trafficking decisions that occur within minutes of bacterial uptake. Mol. Microbiol. 28, 663-674. doi: 10.1046/j.13652958.1998.00841.x

Sadosky, A. B., Wiater, L. A., and Shuman, H. A. (1993). Identification of Legionella pneumophila genes required for growth within and killing of human macrophages. Infect. Immun. 61, 5361-5373.

Santic, M., Asare, R., Doric, M., and Abu Kwaik, Y. (2007). Host-dependent trigger of caspases and apoptosis by Legionella pneumophila. Infect. Immun. 75, 2903-2913. doi: 10.1128/IAI.00147-07

Scharf, J. M., Damron, D., Frisella, A., Bruno, S., Beggs, A. H., Kunkel, L. M., et al. (1996). The mouse region syntenic for human spinal muscular atrophy lies within the Lgn 1 critical interval and contains multiple copies of Naip exon 5. Genomics 38, 405-417. doi: 10.1006/geno.1996.0644

Segal, G., Purcell, M., and Shuman, H. A. (1998). Host cell killing and bacterial conjugation require overlapping sets of genes within a $22-\mathrm{kb}$ region of the Legionella pneumophila genome. Proc. Natl. Acad. Sci. U.S.A. 95, 1669-1674. doi: 10.1073/pnas.95.4.1669

Silveira, T. N., and Zamboni, D. S. (2010). Pore formation triggered by Legionella spp. is an Nlrc4 inflammasome-dependent host cell response that precedes pyroptosis. Infect. Immun. 78, 1403-1413. doi: 10.1128/IAI.00905-09

Spörri, R., Joller, N., Albers, U., Hilbi, H., and Oxenius, A. (2006). MyD88dependent IFN-gamma production by NK cells is key for control of Legionella pneumophila infection. J. Immunol. 176, 6162-6171.
Srinivasula, S. M., Poyet, J.-L., Razmara, M., Datta, P., Zhang, Z., and Alnemri, E. S. (2002). The PYRIN-CARD protein ASC is an activating adaptor for caspase-1. J. Biol. Chem. 277, 21119-21122. doi: 10.1074/jbc.C200179200

Thornberry, N. A., Bull, H. G., Calaycay, J. R., Chapman, K. T., Howard, A. D., Kostura, M. J., et al. (1992). A novel heterodimeric cysteine protease is required for interleukin-1 beta processing in monocytes. Nature 356, 768-774. doi: $10.1038 / 356768 \mathrm{a} 0$

Vance, R. E., Isberg, R. R., and Portnoy, D. A. (2009). Patterns of pathogenesis: discrimination of pathogenic and nonpathogenic microbes by the innate immune system. Cell Host Microbe 6, 10-21. doi: 10.1016/j.chom.2009. 06.007

Vinzing, M., Eitel, J., Lippmann, J., Hocke, A. C., Zahlten, J., Slevogt, H., et al. (2008). NAIP and Ipaf control Legionella pneumophila replication in human cells. J. Immunol. 180, 6808-6815.

Vogel, J. P., Andrews, H. L., Wong, S. K., and Isberg, R. R. (1998). Conjugative transfer by the virulence system of Legionella pneumophila. Science 279, 873-876. doi: 10.1126/science.279.5352.873

Wang, S., Miura, M., Jung, Y. K., Zhu, H., Gagliardini, V., Shi, L., et al. (1996). Identification and characterization of Ich-3, a member of the interleukin-1beta converting enzyme (ICE)/Ced-3 family and an upstream regulator of ICE. J. Biol. Chem. 271, 20580-20587. doi: 10.1074/jbc.271.34. 20580

Wang, S., Miura, M., Jung, Y. K., Zhu, H., Li, E., and Yuan, J. (1998). Murine caspase-11, an ICE-interacting protease, is essential for the activation of ICE. Cell 92, 501-509. doi: 10.1016/S0092-8674(00)80943-5

Wright, E. K., Goodart, S. A., Growney, J. D., Hadinoto, V., Endrizzi, M. G., Long, E. M., et al. (2003). Naip5 affects host susceptibility to the intracellular pathogen Legionella pneumophila. Curr. Biol. 13, 27-36. doi: 10.1016/S09609822(02)01359-3

Yang, J., Zhao, Y., Shi, J., and Shao, F. (2013). Human NAIP and mouse NAIP1 recognize bacterial type III secretion needle protein for inflammasome activation. Proc. Natl. Acad. Sci. U.S.A. 110, 14408-14413. doi: 10.1073/pnas. 1306376110

Zamboni, D. S., Kobayashi, K. S., Kohlsdorf, T., Ogura, Y., Long, E. M., Vance, R. E., et al. (2006). The Bircle cytosolic pattern-recognition receptor contributes to the detection and control of Legionella pneumophila infection. Nat. Immunol. 7, 318-325. doi: 10.1038/ni1305

Zhao, Y., Yang, J., Shi, J., Gong, Y.-N., Lu, Q., Xu, H., et al. (2011). The NLRC4 inflammasome receptors for bacterial flagellin and type III secretion apparatus. Nature 477, 596-600. doi: 10.1038/nature10510

Conflict of Interest Statement: The authors declare that the research was conducted in the absence of any commercial or financial relationships that could be construed as a potential conflict of interest.

Received: 21 August 2013; accepted: 16 December 2013; published online: 27 December 2013.

Citation: Casson CN and Shin S (2013) Inflammasome-mediated cell death in response to bacterial pathogens that access the host cell cytosol: lessons from Legionella pneumophila. Front. Cell. Infect. Microbiol. 3:111. doi: 10.3389/fcimb.2013.00111

This article was submitted to the journal Frontiers in Cellular and Infection Microbiology.

Copyright (c) 2013 Casson and Shin. This is an open-access article distributed under the terms of the Creative Commons Attribution License (CC BY). The use, distribution or reproduction in other forums is permitted, provided the original author(s) or licensor are credited and that the original publication in this journal is cited, in accordance with accepted academic practice. No use, distribution or reproduction is permitted which does not comply with these terms. 